\title{
SISTEMAS TAMPÃO: UMA ESTRUTURA DIDÁTICA TEÓRICO-PRÁTICA
}

Bianca Caroline Rossi-Rodrigues", Elaine Aparecida de Oliveira e Eduardo Galembeck

Departamento de Bioquímica, Instituto de Biologia, Universidade Estadual de Campinas, 13083-970 Campinas - SP, Brasil

Recebido em 28/2/08; aceito em 17/9/08; publicado na web em 15/4/09

\begin{abstract}
BUFFERING-SYSTEMS: A THEORETICAL AND PRACTICAL DIDATIC APPROACH. Courses with practical and theoretical classes are potentially didactic structures for the development of motor and complex cognitive skills when operated in an integrated manner. When worked aligned, theory and practice enable a clearer view of the issue, opening scope for introducing concepts from other disciplines and for the development of lab skills. This paper presents a methodology for biochemistry labwork that promotes the teaching of the buffering systems contents together with to the development of scientific research practice concepts in an interdisciplinary perspective that integrates theory and practice.
\end{abstract}

Keywords: practical class methodology; labwork; buffering-systems.

Este artigo descreve uma atividade prática sobre sistemas tampão, propondo uma estrutura didática que una a aprendizagem de conteúdo teórico, ou conceituação abstrata, ao desenvolvimento de conceitos sobre a prática científica, ou seja, integrando teoria e prática em uma abordagem investigativa.

Em disciplinas de Química, o contexto de aulas práticas refere-se àquelas que ocorrem em um laboratório, utilizando vidrarias, reagentes e equipamentos específicos. São desenvolvidas numa dinâmica particular de acordo com o assunto tratado, a disponibilidade da infra-estrutura do local e da equipe docente e seus auxiliares.

Muitas habilidades podem ser desenvolvidas nas aulas práticas, desde a manipulação de equipamentos, reagentes e vidrarias em um contexto laboratorial, dentro de padrões de segurança, ${ }^{1,2}$ bem como as atitudes requeridas para que seja realizada uma pesquisa científica. ${ }^{3,4}$

A American Society for Biochemistry and Molecular Biology (ASBMB) listou uma série de habilidades e capacidades que os estudantes devem desenvolver até o final de um programa de Bioquímica e Biologia Molecular, sendo a maioria delas adquirida em aulas práticas. ${ }^{2}$ No entanto, uma importante função destas, muito trabalhada nas disciplinas de Bioquímica, é a de ilustrar conceitos teóricos. ${ }^{5}$

Atividades práticas e teóricas integradas, ao menos no que se refere ao conteúdo, proporcionam um novo ângulo de visão do assunto, permitindo que o estudante verifique a origem de um determinado conceito ou quais dados experimentais permitiram formular uma determinada teoria, ou até mesmo qual é a utilidade de um dado experimento para a sociedade. Os conceitos se ampliam: saem do livro e podem chegar a se integrar na vida diária do estudante.

Ainda que possua potencial para o desenvolvimento de processos cognitivos, as aulas práticas de Bioquímica, muitas vezes, são reduzidas ao desenvolvimento de habilidades motoras, enfocando a instrumentação, onde o objetivo passa a ser obter resultados precisos e repetitivos a partir de protocolos pré-estabelecidos. Os estudantes não são provocados a fazer a conexão entre os conteúdos apresentados em aulas expositivas com os experimentos realizados em laboratório.

Neste contexto, apresentamos a proposta de uma atividade aplicada em disciplinas de Bioquímica, realizadas nos primeiros anos de graduação. Tal atividade envolve a instrumentação laboratorial, o desenvolvimento de habilidades motoras e de habilidades cognitivas complexas através das quais o estudante entrará em contato com o mé-

*e-mail: rossirodriguesbc@gmail.com todo científico de pesquisa experimental, podendo verificar implicações do método, num ambiente que associa intimamente teoria e prática.

\section{O TEMA SISTEMAS TAMPÃO EM DISCIPLINAS DE BIOQUÍMICA}

Estudos revelam que estudantes de graduação enfrentam dificuldades em relacionar fenômenos macroscópicos com os processos microscópicos envolvidos. ${ }^{6}$ Acredita-se que o entendimento dos sistemas tampão resume-se à resolução de equações matemáticas. ${ }^{7}$ A abordagem de sistemas tampão como conteúdo introdutório em disciplinas de Bioquímica permite a retomada de conceitos vistos em Química e a construção da compreensão dos processos metabólicos e, sob um olhar macroscópico, fisiológico, alicerçados e integrados ao entendimento dos conceitos sobre sistemas tampão e sua importância.

\section{PROPOSTA DA ATIVIDADE TEÓRICO-PRÁTICA SOBRE SISTEMAS TAMPÃO}

As aulas práticas da disciplina de Bioquímica (BB280) para os cursos de Ciências Biológicas, do Instituto de Biologia da Unicamp, vêm passando por uma série de reformulações nos últimos anos, deixando de ter um enfoque excessivo em atividades de instrumentação e demonstração.

A atividade sobre Sistemas Tampão da matéria referida foi a primeira a ser reformulada, passando a ser baseada no confronto do modelo teórico, abstrato, com os resultados experimentais, abordado através da comparação entre a simulação computacional e a respectiva experimentação no laboratório. É simulada uma titulação e, na aula seguinte, a titulação é executada em laboratório, de acordo com os parâmetros da simulação (tipo de tampão e titulantes, concentrações e volumes). A atividade tem como foco a aprendizagem da metodologia da pesquisa científica - entendimento de como planejar um experimento, recolher dados, interpretar resultados e confeccionar um relatório - bem como a aprendizagem de conteúdo e o reconhecimento das dificuldades e possibilidades da prática laboratorial.

Em sua atual estrutura, a atividade é composta por 2 aulas em laboratório (com duração de $4 \mathrm{~h}$ cada), ministradas após a aula teórica sobre o tema. Os alunos são organizados em grupos de, no máximo, 6 pessoas. 


\section{Desenvolvimento da atividade}

Na primeira aula, os estudantes respondem às questões (de "a" até "g") do Estudo Dirigido (ED) (Quadro 1), realizam a simulação e planejam a experimentação da aula seguinte.

A simulação reproduz uma titulação no computador, usando programas como o Microsoft Excel ou OpenOffice. São estabelecidos os parâmetros que serão reproduzidos na aula seguinte, ministrada em laboratório. Com o uso do modelo matemático (HendersonHasselbach) e cálculos de diluição é determinada a faixa tamponante do sistema em questão pela simulação da adição de $\mathrm{HCl}$ e $\mathrm{NaOH}$. Com o auxílio do professor e dos monitores, cada grupo cria uma planilha (Figura 1), organizando e inserindo as equações matemáticas necessárias para tal simulação.

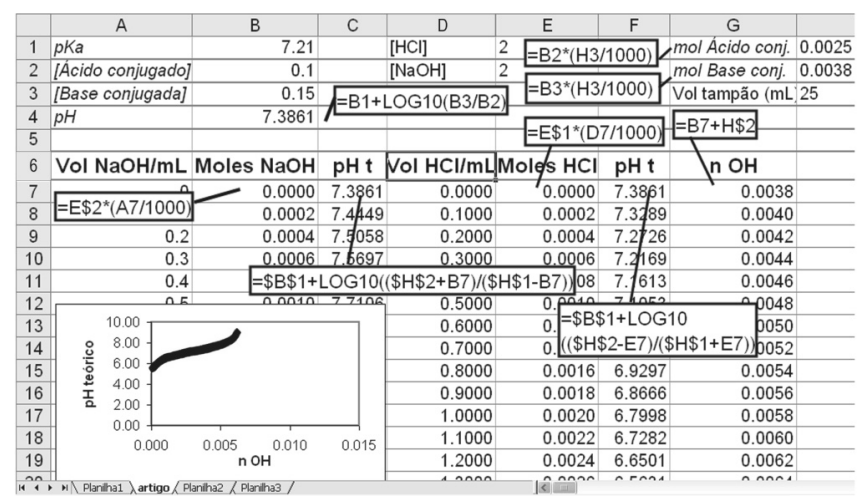

Figura 1. Planilha de simulação da titulação preparada pelos alunos no laboratório de informática durante a primeira aula da atividade. As caixas de texto indicam as fórmulas utilizadas

As primeiras informações fornecidas para o início dos cálculos contêm os dados das soluções que serão disponibilizadas na aula seguinte para a execução da titulação no laboratório. É fornecida a concentração do ácido e da base conjugados que formarão o tampão (Figura 1, B2 e B3) e o pH do tampão (Figura 1, B1), conforme o roteiro da atividade. É sugerida uma solução de titulantes $(\mathrm{HCl}$ e $\mathrm{NaOH} 2 \mathrm{M}$ ) (Figura 1, E1 e E2) e o volume final dos mesmos (Figura 1, H4). Esses parâmetros sugeridos podem ser modificados durante a simulação para a definição das melhores condições de titulação. Todos os outros parâmetros serão calculados através das equações matemáticas. Um grupo de monitores e o professor acompanham os grupos de alunos, auxiliando-os com os cálculos.

Durante a etapa de planejamento os alunos listam no caderno de laboratório, sucintamente, todos reagentes e vidrarias que serão necessários (questão h do ED).

Na segunda aula, é realizado no laboratório o procedimento experimental antecipadamente modelado no computador, seguindo o planejamento e usando os mesmos volumes e reagentes estipulados nos cálculos da simulação da titulação.

\section{A ATIVIDADE DE SISTEMAS TAMPÃO E O DESENVOLVIMENTO DE HABILIDADES}

Nesta sessão, discutiremos as habilidades desenvolvidas na atividade, em que contexto foram trabalhadas e quais vias didáticas foram percorridas para alcançá-las.

\section{Promover uma visão integrada sobre teoria e prática}

Aulas práticas e teóricas possuem uma estrutura muito diferenciada. No geral, um mesmo conteúdo demanda um tempo maior para ser desenvolvido em uma aula prática. Por isso, em muitos casos, esses dois aspectos são ministrados em momentos diferentes na disciplina, ainda que digam respeito a um mesmo assunto, o que gera um distanciamento temporal entre prática e teoria. Em algumas disciplinas também se verifica o distanciamento temático entre os mesmos. Os dois tipos de aula não são planejados em conjunto, o que dificulta uma ligação direta entre ambas, ligação esta essencial para um bom aproveitamento e para a aprendizagem do assunto. Essa distância (temática e temporal) entre a parte experimental e a conceituação abstrata que a fundamenta é um dos grandes fatores que desmotivam os estudantes nas disciplinas de Bioquímica. ${ }^{8}$ Estudos sobre a preferência do tipo de ambiente laboratorial mostram que os estudantes preferem aulas integradas a aulas tradicionais. ${ }^{9}$ As aulas teóricas devem proporcionar a base para as aulas práticas, já que o domínio da teoria é fator fundamental para um bom planejamento e essencial para a interpretação dos dados experimentais. ${ }^{10}$ Desse modo, a integração entre estas possibilita que aluno veja a Bioquímica de uma maneira mais ampla. ${ }^{1}$

É nesse contexto, então, que a atividade de sistemas tampão é estruturada. Em sua primeira aula, um Estudo Dirigido(ED) retoma conceitos puramente teóricos, passando gradativamente para o teórico-prático.

As primeiras questões (a e b, no anexo 1) abordam conceitos de ionização de ácidos e constantes de equilíbrio. Na questão (c), os alunos montam a equação de Henderson-Hasselbach com dados do tampão que será utilizado. Conceitos de diluição também são trabalhados no $\mathrm{ED}$, com a simulação do preparo de um tampão a partir de soluções estoque (questão d).

Até esse momento, as questões possuem uma fundamentação abstrata, mas precisarão ser utilizadas para dar prosseguimento ao ED que passa a apresentar um caráter teórico-prático nas próximas questões (f, g e i), através do desenvolvimento do modelo matemático.

A segunda parte do ED já possui um caráter prático. Realizada no laboratório, trata-se do momento da execução da experimentação, baseada nos dados do modelo teórico e no planejamento desenvolvidos na aula anterior.

O confronto entre o modelo abstrato e os resultados empíricos, trabalhados sob uma estrutura integrada, permite mais que reproduzir um procedimento experimental, pois reconstrói um conceito alicerçado numa visão alimentada pela teoria e pela experimentação.

Dentro dessa situação integrada, vias didáticas interdisciplinares permitem o desenvolvimento de cognição complexa. ${ }^{11}$ A matemática e a computação, utilizadas como ferramentas de simulação, estimulam a abstração do tema, retomando conhecimentos da memória, adquiridos em outro contexto. ${ }^{10}$ Os mesmos estudos revelam que o trabalho com softwares computacionais anterior à prática laboratorial de química proporciona uma visão prévia da experiência laboratorial, permitindo reconhecer aspectos de importância primária na atividade, otimizando-a e aumentando o desempenho do aluno durante a prática laboratorial. ${ }^{10}$

A investigação científica, na grande maioria das disciplinas, requer um mínimo de conhecimento matemático, seja para o entendimento e desenvolvimento de gráficos ou para a compreensão de modelos estatísticos. Muito dos conhecimentos de química estão alicerçados em cálculos matemáticos. ${ }^{10}$ No entanto, a literatura e a prática diária revelam deficiências em conceitos matemáticos em estudantes de química. ${ }^{8}$ Percebe-se desde dificuldades em construir e interpretar gráficos até a compreensão de equações algébricas. Estimular o pensamento matemático durante as aulas de disciplinas de Bioquímica contribui, portanto, para a aprendizagem de conceitos baseada em uma perspectiva interdisciplinar.

\section{Introdução à metodologia de pesquisa científica}

Planejar, executar e coletar dados, assim como sintetizar e desenvolver um relatório são pilares que sustentam a pesquisa de um trabalho científico. Tal investigação pode fazer aflorar habilidades do 


\section{Quadro 1}

\section{Roteiro da atividade de Sistemas Tampão da Disciplina Bioquímica Básica (BB280) - Unicamp, 2006-2007}

Planejamento e Execução dos Experimentos

\section{Sistemas Tampão - Planejamento de Experimentos (ED)}

Grupo 1

Considerando um volume de $500 \mathrm{~mL}$ de uma solução-tampão formada de $\mathrm{NaH}_{2} \mathrm{PO}_{4(\text { aq })} 0,100 \mathrm{M} \mathrm{e} \mathrm{Na}_{2} \mathrm{HPO}_{4(\text { aq })} 0,100 \mathrm{M}$.

a) Escreva a equação de todas as etapas de ionização do $\mathrm{H}_{3} \mathrm{PO}_{4}$ em água.

b) Escreva a equação da constante de equilíbrio para cada uma das ionizações do ácido em água.

c) Escreva a equação de Henderson-Hasselbalch para o tampão em questão e diga qual o valor de pKa que deverá ser utilizado.

d) Mostre os cálculos necessários para preparar o tampão a partir das soluções estoque fornecidas.

e) Calcule o pH teórico da solução tampão.

f) Qual será o valor do $\mathrm{pH}$ teórico resultante da adição de $0,8 \mathrm{~mL}$ de $\mathrm{NaOH} 1 \mathrm{M}$ em $15 \mathrm{~mL}$ de tampão?

g) Qual será o valor do pH teórico resultante da adição de $0,8 \mathrm{~mL}$ de $\mathrm{HCl} 1 \mathrm{M}$ em $20 \mathrm{~mL}$ de tampão?

h) Liste os equipamentos e vidraria necessários para realizar a titulação da solução tampão fornecida (Utilizando os itens disponíveis no armário da bancada. Caso necessite de algum item não disponível na bancada consultar os monitores sobre a disponibilidade do mesmo.).

i) Calcule os valores teóricos de $\mathrm{pH}$ a cada adição de ácido e de base à solução tampão preparado (definir o volume de tampão (titulado) e concentração dos titulantes (ácido e base) que serão utilizados).

j) Seguindo o modelo abaixo, complete as tabelas a partir dos cálculos realizados.

\begin{tabular}{|c|c|c|c|c|c|c|}
\hline Volume de $\mathrm{NaOH}(\mathrm{mL})$ & $\mathrm{nNaOH}$ & $\mathrm{pH}_{\text {teórico }}$ & $\mathrm{pH}_{\text {experimental } 1}$ & $\mathrm{pH}_{\text {experimental 2 }}$ & $\mathrm{pH}_{\text {experimental } 3}$ & média pH experimental \\
\hline Volume de $\mathrm{HCl}(\mathrm{mL})$ & $\mathrm{nHCl}$ & $\mathrm{pH}_{\text {teórico }}$ & $\mathrm{pH} \mathrm{experimental} 1$ & $\mathrm{pH}$ experimental 2 & $\mathrm{pH}$ experimental 3 & média pH experimental \\
\hline \multicolumn{7}{|l|}{ Dado: } \\
\hline Ácido & $\mathrm{Ka}_{1}$ & $\mathrm{pKa}_{1}$ & $\mathrm{Ka}_{2}$ & $\mathrm{pKa}_{2}$ & $\mathrm{Ka}_{3}$ & $\mathrm{pKa}_{3}$ \\
\hline Ácido fosfórico $\mathrm{H}_{3} \mathrm{PO}_{4}$ & $7,6 \times 10^{-3}$ & 2,12 & $6,2 \times 10^{-8}$ & 7,21 & $2,1 \times 10^{-13}$ & 12,68 \\
\hline
\end{tabular}

Soluções disponíveis para o experimento:

Solução estoque de $\mathrm{NaOH} \cong 5 \mathrm{M}$ (a concentração exata será fornecida na aula);

Solução estoque de $\mathrm{HCl} 5 \mathrm{M}$;

Solução estoque de $\mathrm{NaH}_{2} \mathrm{PO}_{4} 1 \mathrm{M}$;

Solução estoque de $\mathrm{Na}_{2} \mathrm{HPO}_{4} 1 \mathrm{M}$;

Sistemas Tampão - Execução de Experimentos (ED)

A parte experimental deverá ser realizada a partir dos cálculos preparados no estudo dirigido

a) Prepare a solução tampão mencionada no estudo dirigido a partir das mesmas soluções estoque fornecidas.

b) Com o uso de um pHmetro, faça a leitura do $\mathrm{pH}$ da solução e compare com o valor teórico. Se necessário, ajuste o pH para o valor esperado de acordo com os cálculos.

c) Com o uso de um pHmetro, faça a leitura do pH da solução tampão após a adição de $0,8 \mathrm{~mL}$ de $\mathrm{NaOH} 1 \mathrm{M}$ em $15 \mathrm{~mL}$ de tampão? Compare esse valor com o valor teórico.

d) Com o uso de um pHmetro, faça a leitura do pH da solução tampão após a adição 0,8 mL de $\mathrm{HCl} 1 \mathrm{M}$ em $20 \mathrm{~mL}$ de tampão? Compare esse valor com o valor teórico.

e) Titule a solução tampão (volume definido na aula anterior), utilizando os mesmos volumes e concentração de ácido e de base utilizados nos cálculos teóricos. Insira os dados na tabela comparando os $\mathrm{pH}$ teóricos e experimentais.

\section{Relatório}

Apresente um relatório segundo as normas exigidas, contendo todos os cálculos realizados.

Apresentar os dados referentes aos valores de $\mathrm{pH}_{\text {teórico }}$ e $\mathrm{pH}_{\text {experimental }}$ em forma de tabela.

Realizar as medidas em triplicata.

Calcular a média dos resultados experimentais.

Apresentar um único gráfico com os resultados teóricos e experimentais. 
processo cognitivo como também habilidades motoras. ${ }^{8,10}$ A proposta de desenvolver o estudo introdutório sobre as diretrizes de uma pesquisa científica responde à preocupação de desenvolver habilidades que estimulem os processos cognitivos de conhecer, compreender, aplicar, analisar e sintetizar.

A seguir será discutido como cada habilidade relevante (planejar, coletar dados, sintetizar e relatar) foi trabalhada e quais as diretrizes didáticas que levaram ao desenvolvimento das mesmas. O processo de execução de experimentos será abordado posteriormente na discussão sobre a percepção das implicações envolvidas na prática laboratorial.

\section{Planejamento de experimentos}

A atividade de planejamento é realizada com o Estudo Dirigido dentro da primeira aula e propõe a determinação e listagem dos materiais a serem utilizados na experimentação da segunda aula. A bancada laboratorial de cada grupo contém uma lista com os materiais ali disponíveis. É necessária a análise desses itens, constatando apenas quais materiais não estão no armário da bancada. Ao pensarem sobre materiais a serem utilizados, os alunos precisam projetar a experimentação, pensando a teoria em um campo ainda desconhecido (o laboratório), determinando como será feita a experimentação, os procedimentos a serem realizados. É necessário também que associem os conceitos abstratos, os dados simulados na planilha eletrônica, as informações do roteiro de laboratório e que simulem mentalmente, naquele instante, a aula seguinte. Atividades pré-laboratoriais preparam o estudante e conferem melhor qualidade à experimentação. ${ }^{12}$ Pesquisas propõem o estímulo ao uso de simulação em aulas práticas, dado que o processo de simular um experimento complementa o entendimento do fenômeno em si e ajuda no desenvolvimento das habilidades de resolução dos problemas envolvidos com o assunto, na tomada de decisões e na observação de resultados. ${ }^{9}$

Esses processos remetem a uma pequena parte da rotina de um pesquisador que necessita de um planejamento diário e rigoroso para a execução da rotina laboratorial, além de contribuírem para a organização e otimização da execução na aula seguinte.

Através de alguns relatos de grupos pôde-se verificar a constatação da importância de se realizar um bom planejamento, como:

“Com relação às dificuldades encontradas para realização do experimento (...) não se planejou completamente todos os possíveis instrumentos a serem utilizados, principalmente a capacidade volumétrica dos mesmos." (trecho do relatório, Grupo A, ${ }^{13}$ BB280 - 2007).

\section{Coletar dados, sintetizar e confeccionar relatório}

Os alunos coletarão dados no caderno de laboratório. É proposta uma organização do caderno em que os grupos anotarão as etapas desenvolvidas em toda a atividade, que abrange: os dados recolhidos no computador, o planejamento, os dados da execução, as etapas ocorridas durante a execução e os possíveis erros e falhas. Essa forma permite que os alunos percebam a importância do registro na pesquisa, pois é o que restará após a experimentação e de onde virão os dados para a discussão. Dados mal coletados podem levar à perda de todo o trabalho. Consideram-se "bons dados experimentais" aqueles que verificam os previstos na simulação. ${ }^{4} \mathrm{Na}$ confecção do relatório, os alunos poderão observar a qualidade das informações que recolheram. Mais à frente discutiremos a percepção das dificuldades referentes ao trabalho laboratorial, que podem levar à não-obtenção de um resultado final esperado.

Os grupos desenvolveram os relatórios com caráter de relatório científico e com estruturas e regras pré-definidas pelo professor, seguindo sempre os moldes de formatação e redação científica.

\section{Desenvolver a percepção sobre as implicações envolvidas na} prática laboratorial

Sob o olhar construtivista, o conhecimento se constrói pela interação com o ambiente, ${ }^{8}$ em outras palavras, o aluno aprende ao agir, fazer, acionar. Em alunos iniciantes na experimentação laboratorial, algumas habilidades em Bioquímica, por mais que sejam explicadas e elucidadas teoricamente, só serão aprendidas efetivamente durante a execução do trabalho laboratorial. Alguns exemplos delas são: as motoras e a visão sobre o processo experimental, ou seja, o reconhecimento das limitações de um equipamento; a do manuseio de vidraria, os imprevistos advindos de erros acidentais, a importância da organização e anotação organizada dos dados. A falta de conhecimento técnico e experiência laboratorial é freqüentemente a causa de não se alcançar o resultado esperado de um experimento. ${ }^{4}$

O confronto entre um modelo teórico com o resultado experimental permite um olhar mais cuidadoso sobre possíveis causas que levaram ao resultado esperado ou não.

A deficiência de habilidade técnica é uma causa mencionada por diversos grupos para não obterem os resultados esperados, como é observado nos trechos de discussão apresentados nos relatórios:

\begin{abstract}
"Durante a primeira medição verificou-se um $\mathrm{pH}$ igual a 6,82. A diferença entre o pH esperado $(7,21)$ e o obtido $(6,82)$ pode ter ocorrido devido a erros nas concentrações das soluções utilizadas elou na medição do volume dos reagentes." (trecho do relatório, Grupo E, BB280 - 2007).

"O distanciamento dos pontos no fim da titulação pode ser explicado por uma questão prática: a adição de 0,1 mL pode não ter sido fiel, ou seja, pode não ter sido adicionado exatamente esta quantidade em alguns pontos da titulação." (trecho do relatório, Grupo D, BB280 - 2007).

"Uma possível explicação para a diferença na estrutura das curvas é que, além do fato do pH inicial ter sido diferente, volumes não tão exatos de ácido ou base podem ter sido adicionados na titulação, devido à imprecisão no momento do experimento para o preenchimento da pipeta" (trecho do relatório, Grupo F, BB280 - 2007).

"A diferença do valor calculado e o valor prático deve-se $\underline{a}$ ineficácia em se medir quantidades exatas dos reagentes envolvidos." (trecho do relatório, Grupo H, BB280 - 2007).
\end{abstract}

Esses grupos referem-se a possíveis erros que podem ter sido cometidos no trabalho com pipetas, provetas, balão volumétrico. Um grupo apontou a questão da precisão como item conclusivo do relatório, mostrando que esse aspecto foi reconhecido como uma implicação importante no contexto do trabalho experimental:

“... a variação do pH teórico e experimental é pequena, apesar de apresentar valores diferentes devido, principalmente, à imprecisão nos volumes a serem titulados. Pôde-se assim concluir que para a realização de um procedimento exato, extremamente semelhante aos cálculos matemáticos, é necessário ter muita precisão. Além disso, é importante ressaltar que o peagâmetro utilizado só possuía duas casas decimais, ou seja, os valores teórico e prático não puderam ser comparados perfeitamente." (trecho do relatório, Grupo F, BB280 - 2007).

Autores apontam que a habilidade de instrumentação está relacionada às habilidades de observação, de conhecer os procedimentos de segurança e técnicas específicas. ${ }^{12}$ As habilidades motoras, portanto, mais do que a proporcionar a elaboração do movimento correto de 
manipulação, estão intimamente associadas ao desenvolvimento de habilidades cognitivas, o que favorece um ambiente produtivo de aprendizagem.

O reconhecimento das limitações de equipamentos, ou de não se utilizar o equipamento que melhor desempenharia uma determinada função também foi item de discussão:

"O peagâmetro possuía precisão de $96 \%$ e apresentava valores com apenas uma casa decimal, o que impedia, por exemplo, a obtenção de valores mais exatos." (trecho do relatório, Grupo D, BB280 - 2007).

"Uma outra provável explicação (de não se atingir o resultado esperado) é que não foi medida e homogeneizada corretamente a mistura, já que não foi usado o agitador magnético o qual conferiria uma mistura mais igualitária..." (trecho do relatório, Grupo F, BB280 - 2007).

O resultado não pôde ser alcançado, muitas vezes, também, pelas propriedades das substâncias trabalhadas, podendo haver comportamentos inesperados em virtude da falta de conhecimento sobre propriedades das substâncias utilizadas:

"A temperatura não foi levada em conta durante a prática e também na realização dos cálculos teóricos; importante lembrar que a temperatura influencia na constante de equilíbrio da reação, e uma constante diferente, implicaria a obtenção de valores diferentes em todos os cálculos efetuados". (trecho do relatório, Grupo D, BB280 - 2007).

"A diferença apresentada entre os resultados obtidos e os esperados pode ter ocorrido pelo fato de que, provavelmente, o $\mathrm{CO}_{2}(\mathrm{~g})$ atmosférico, entrando em contato com a solução aquosa, formou ácido carbônico $\left(\mathrm{H}_{2} \mathrm{CO}_{3}(\mathrm{aq})\right)$. (...) Outro fator que possivelmente poderia explicar tal diferença é a volatilidade das soluções, o que aumentaria a concentração da base adicionada ao sistema tampão e assim o ácido conjugado teria um efeito menos intenso." (trecho do relatório, Grupo E, BB280 - 2007).

"No caso do nosso experimento, os resultados podem ter sido afetados por alguns imprevistos durante a realização, como a precipitação da solução estoque de $\mathrm{Na}_{2} \mathrm{HPO}_{4}$. Na solução final do tampão, também foram percebidas partículas em suspensão." (trecho do relatório, Grupo M, BB280 - 2007).

A busca por justificativas para a discussão sobre imprevistos advindos do comportamento das substâncias induz os alunos à retomada de conceitos teóricos, associando-os e articulando-os para o entendimento da questão. Contribui também para reforçar o aprendizado desses conceitos através de uma visão integrada da questão e para a observação de que a Bioquímica não é uma disciplina isolada, mas depende do entendimento de conteúdos desenvolvidos, principalmente, em disciplinas de Química Geral.
$\mathrm{O}$ fato de alguns grupos terem se sentido inseguros quanto a ter ocorrido determinada falha ou acidente demonstra a falta de cuidados na observação desses parâmetros e, provavelmente, deve tê-los tornado mais alertas para situações análogas, em experimentos subseqüentes.

\section{CONCLUSÃO}

O modelo estrutural de aulas práticas desenvolvido representa uma via didática que permite o ensino do conteúdo de sistemas tampão de maneira ampla, abrangendo o trabalho do conteúdo teórico em consonância com o desenvolvimento de habilidades advindas de um contexto empírico.

A estrutura de aproximação da prática à conceituação abstrata que a sustenta possibilita um caráter interdisciplinar, abrangendo conceitos matemáticos, computacionais e químicos.

A abordagem teórico-prática possibilita aos estudantes uma percepção sobre as implicações envolvidas na prática laboratorial pelo reconhecimento de possíveis causas que podem levar ao sucesso ou insucesso do resultado esperado. Entre as possíveis causas de não se alcançar o resultado esperado, observa-se a deficiência de habilidade técnica para realizar a execução laboratorial, verificada por erros experimentais, limitações de equipamentos, desconhecimento das propriedades das substâncias trabalhadas, verificado pelo comportamento inesperado das mesmas durante a experimentação.

$\mathrm{O}$ domínio das bases teóricas referentes às atividades práticas e o confronto de dados teóricos com experimentais aparecem nas discussões dos relatórios de maneira bastante rica, indicando um nível de compreensão dos conteúdos bastante satisfatório.

\section{REFERÊNCIAS E NOTAS}

1. Kotiw, M.; Learmonth, P.; Sutherland, M. W.; Biochemical Education 1999, 27, 131

2. http://www.asbmb.org, acessada em Janeiro 2008.

3. Wood, E.J.; Biochemical Education 1996, 24, 132.

4. Pich-Otero, A.; Molina-Ortiz, S.; Delaplace, L.; Castellani, O.; Hozbor, D.; Sorgentni, D.; Lodeiro, A.; Biochem. Educ. 1998, 26, 281.

5. Alvarez, M. A.; Tese de Doutorado, Universidade de São Paulo, Brasil, 2002.

6. Orgill, M.; Sutherland, A.; Chemistry Education Research and Practice 2008, 9, 131.

7. White, H. B.; Biochem. Mol. Biol. Educ. 2005, 33, 54.

8. Potgieter, M.; Harding, A.; Engelbrecht, J.; Journal of Research in Science Teaching 2007, 45, 197

9. Hofstein, A.; Lunetta, V. N.; Science Education 2004, 88, 28.

10. Bangash, F. K.; Proceddings of 10a IOST International Organization for Science and Technology Education, Foz do Iguaçu, Brasil, 2002.

11. Bel, E.; Biochem. Mol. Biol. Educ. 2003, 31, 225.

12. Reid, N.; Shah, I.; Chemistry Education Research and Practice 2007, 8, 172.

13. Os grupos foram discriminados aleatoriamente por letras, não representando sua real denominação. 